\title{
Italique
}

Poésie italienne de la Renaissance

XVII | 2014

Varia

\section{Il sogno erotico nella lirica del Cinquecento}

\section{Erika Milburn}

\section{OpenEdition}

\section{Journals}

Edizione digitale

URL: https://journals.openedition.org/italique/384

DOI: 10.4000/italique.384

ISSN: 1663-4438

\section{Editore}

Librairie Droz

\section{Edizione cartacea}

Data di pubblicazione: 1 octobre 2014

Paginazione: 43-71

ISBN: 978-2-600-01841-8

ISSN: 1423-3983

\section{Notizia bibliografica digitale}

Erika Milburn, «Il sogno erotico nella lirica del Cinquecento», Italique [Online], XVII | 2014, online dal 01 octobre 2016, consultato il 21 septembre 2021. URL: http://journals.openedition.org/italique/384 ; DOI: https://doi.org/10.4000/italique.384 
ERIKA MiLb U R N

IL SOGNO EROTICO

NELLA LIRICA DEL CIN Q UECENTO 



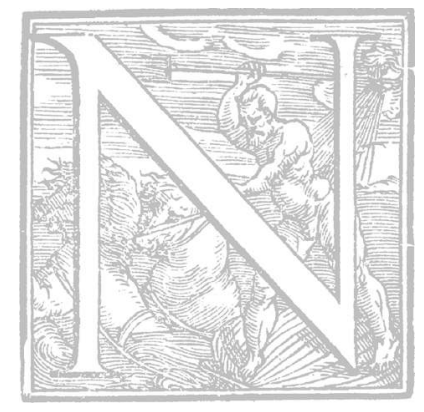

el petrarchismo cinquecentesco il sogno erotico rappresenta uno dei pochi spazi concessi alla sensualità, in un genere nel quale espressioni franche della sessualità e la rappresentazione realistica di atti amorosi sono pressoché assenti. Spostare in ambito onirico queste tematiche rappresenta un escamotage per trattare argomenti generalmente proibiti senza intaccare $i$ cardini del sistema petrarchesco: la castità ed irraggiungibilità dell'amata, il suo ruolo come guida spirituale, la natura non corrisposta ed infelice dell'amore.

Breve storia (letteraria) del sogno erotico tra antichità e medioevo

Prima di passare all'analisi approfondita delle rappresentazioni del sogno erotico nella lirica cinquecentesca sarà utile qualche considerazione sul tema, e sul sogno in generale, nella letteratura classica e medievale.

Secondo gli antichi, $i$ sogni si potevano suddividere in varie categorie, in base alla loro natura (profetica, allegorica, 'messaggiera' ecc.) e provenienza (mandati dagli dèi, scaturiti dalle condizioni fisiologiche o psicologiche del dormiente). A prescindere della precisa classificazione, che varia notevolmente a seconda dell'autore e del periodo storico, una preoccupazione costante era l'attendibilità dei sogni: se cioè erano veri o falsi (in termini poetici se uscivano dalla porta di corno o da quella di avorio);' è importante notare che il concetto di 'falsità' si applicava non soltanto ai sogni 'ingannevoli' ma anche a quelli privi di significato. Quasi tutti coloro che nell' antichità si sono occupati di sogni (professionisti della divinazione, autori di opere storiche o letterarie, filosofi e medici) concordano nell'affermare che $i$ sogni erotici sono 'falsi' in quanto semplici riflessi di desideri presenti anche nello stato di veglia; l'unica significativa eccezione sono $i$ sogni incestuosi, a cui viene attribuito in genere un significato profetico. ${ }^{2}$ Per citare solo un paio di esempi, nella suddivisione dei sogni in cinque tipi proposta da Macrobio nel commento al Somnium Scipionis di Cicerone, quelli dell'amante che gode dell' amata o soffre per la sua assenza vengono classificati tra gli insomnia: 
est enim évú $\pi$ เov quotiens cura oppressi animi corporisve sive fortunae qualis vigilantem fatigaverat talem se ingerit dormienti: animi si amator deliciis suis aut fruentem se videat aut carentem, si metuens quis imminentem sibi vel insidiis vel potestate personam aut incurrisse hanc ex imagine cogitationum suarum aut effugisse videatur. ${ }^{3}$

Anche per Artemidoro, autore di un manuale per l'interpretazione dei sogni, l'Oneirocritica, il sogno insignificante, tra cui quello erotico:

è ciò che è privo di significato e non preannuncia alcun evento futuro, bensì ha efficacia soltanto nel sonno e avviene in seguito a un desiderio irrazionale o a un violento timore, oppure per soverchio peso del ventre o per mancanza di nutrimento. ${ }^{4}$

Va ricordato che ovviamente non tutti $i$ sogni in cui compare la persona amata sono a sfondo sensuale. Ė frequente l'apparizione in sogno dell'amata o dell'amato per dare un messaggio al dormiente: Cinzia si mostra in sogno a Properzio per rivelargli l'identità dei suoi assassini (Eleg. IV 7) come anche il marito di Didone (Aen. I 353-59); Morfeo comunica ad Alcione la morte del marito Ceice in sogno, nelle vesti di quest'ultimo (Met. XI 650-709). I sogni possono avere significati meno precisi, come gli incubi di Didone nell'Eneide (IV 465-68), in cui rivive ripetutamente l'abbandono da parte di Enea, parte di una serie di segni premonitori della propria morte. Per contro, alcuni sogni di persone amate hanno esito positivo: nell'Odissea Penelope vede Ulisse accanto a sé nel letto, un felice presagio del ritorno dell'eroe (XX 83-94).

La mancanza di significato aiuta a spiegare la relativa rarità dei sogni erotici nella letteratura antica.' Nonostante la diffusione di temi affini quali la notte e il sonno nell'elegia romana, il sogno erotico è pressoché assente, forse perché il sonno era considerato nemico degli amanti ed antitetico al ruolo attivo a cui ambiva l'amante maschile, gradito solo se condiviso con la donna ${ }^{7}$ o quando ad assopirsi era il marito dell'amata. ${ }^{8}$ Di conseguenza nella letteratura latina tali sogni sono una prerogativa quasi esclusivamente femminile, 9 espressione di una condizione effettiva e psicologica di sottomissione ed impotenza. ${ }^{\text {Iо }}$

Le rappresentazioni più dettagliate di sogni amorosi si leggono nelle Eroidi di Ovidio. Per le eroine ovidiane (solo in un caso, infatti, è un uomo a vedere in sogno la donna desiderata: Paride) ${ }^{\mathrm{I}}$ il sogno erotico 
svolge un ruolo consolatorio, supplendo all'assenza o all'abbandono dell'amato. Questa funzione viene esplicitata da Laodamia, lasciata sola dal marito Protesilao subito dopo le nozze: "Aucupor in lecto mendaces caelibe somnos; / dum careo veris, gaudia falsa iuvant» (XIII IO5-IO6), con un conciso ma efficace contrasto tra il «lecto caelibe» e $i$ «mendaces somnos», le gioie vere negate dal destino e quelle false del sogno. Questo concetto viene ribadito nel racconto piu circostanziato di Ero, che si sofferma ancora sugli inganni del sogno; altri motivi destinati ad avere una cospicua fortuna nella lirica cinquecentesca sono il lamento contro la brevità del sogno, la speranza che si avveri e l'idea che il sogno possa concedere di godere dell'amato contro la sua volontà:

Forsitan invitus mecum tamen, improbe, dormis et, quamquam non vis ipse venire, venis.

Nam modo te videor prope iam spectare natantem, bracchia nunc umeris umida ferre meis, nunc dare, quae soleo, madidis velamina membris, pectora nunc nostro iuncta fovere sinu multaque praeterea linguae reticenda modestae, quae fecisse iuvat, facta referre pudet.

Me miseram! Brevis est haec et non vera voluptas; nam tu cum somno semper abire soles.

Firmius, o, cupidi tandem coeamus amantes, nec careant vera gaudia nostra fide!

(Ovidio, Eroides, XIX 57-68)

La falsità dei sogni erotici - nonostante l'ora propizia ai sogni veri, vicina all'alba - viene sottolineata implicitamente dal confronto con un altro sogno, questa volta allegorica e profetica, del delfino che si insabbia sulla riva e muore, prefigurando la morte di Leandro (193-204).

Un altro racconto dettagliato di un sogno erotico viene offerto da Saffo, dopo il suo abbandono da parte di Faone:

Tu mihi cura, Phaon; te somnia nostra reducunt, somnia formoso candidiora die.

Illic te invenio, quamvis regionibus absis; sed non longa satis gaudia somnus habet. Saepe tuos nostra cervice onerare lacertos, saepe tuae videor supposuisse meos; oscula cognosco, quae tu committere lingua 
aptaque consueras accipere, apta dare.

Blandior interdum verisque simillima verba eloquor, et vigilant sensibus ora meis.

Ulteriora pudet narrare, sed omnia fiunt, et iuvat, et siccae non licet esse mihi.

At cum se Titan ostendit et omnia secum, tam cito me somnos destituisse queror

(Ovidio, Eroides, Xv I 23-36)

Come nel sogno di Ero (e a differenza di quello di Laodamia), si tratta di una rivisitazione onirica di esperienze già vissute nella vita reale. In omaggio alla pudicizia femminile, il racconto si ferma alla descrizione dei baci scambiati, sorvolando sui dettagli di ciò che segue: espediente che ricorre anche nelle rivisitazioni petrarchistiche del tema in cui la carica erotica viene affidata generalmente a velate allusioni e non, come accade nella poesia erotica romana e neolatina, a descrizioni circostanziate dell'atto sessuale. Come l'epistola di Ero, quella di Saffo fornisce alla lirica cinquecentesca una serie di spunti importanti: il lamento contro la brevità del sogno e l'aurora che lo interrompe; la preferenza, dovuto al piacere provato in sogno, per la notte rispetto al giorno; l'ambiguità del sogno e delle emozioni che provoca, sottolineata dal ricorso costante all' antitesi, al paradosso e a strutture chiastiche; l'implicita condizione di sottomissione di chi sogna, costretta a subire un'assenza o un abbandono. Se nelle Eroidi $i$ sogni erotici delle protagoniste servono principalmente a delineare il loro stato d'animo e ad accentuare il contrasto tra la loro attuale condizione disperata ed una felicità passata o auspicata, nella storia dell'amore incestuoso di Biblide per il fratello Cauno (Met. IX 450-665), il sogno svolge una funzione narrativa fondamentale. È un sogno erotico che per la prima volta rivela a Biblide di essersi innamorata del fratello, spiegando cosi una serie di 'sintomi' misteriosi. Nel lungo soliloquio che segue il risveglio, la ragazza si interroga sul motivo e sulla natura del sogno, combattuta tra la speranza che sia falso e l'auspicio che si avveri; alla fine, incoraggiata da ciò che potrebbe essere una profezia, confessa il suo amore in una lettera. Ma il sogno si rivela falso: Cauno la respinge indignato; Biblide, sconvolta per il rifiuto, impazzisce e infine si trasforma in fonte. Oltre alle tematiche già introdotte nelle Eroidi, appaiono qui alcuni motivi che avranno largo seguito nella poesia italiana: la richiesta che il sogno si ripeti con l'affermazione esplicita che ciò che non è lecito nella vita reale lo è in 
sogno (480-81: «saepe licet simili redeat sub imagine somnus: testis abest somno, nec abest imitata voluptas»); il piacere provato anche solo a ricordare il sogno, nonostante la sua brevità (485: «ut meminisse iuvat! Quamvis brevis illa voluptas»); $i$ dubbi sull'attendibilità del sogno che aprono e chiudono il lungo discorso di Biblide.

In epoca tardo antica e medievale ${ }^{\mathrm{I} 2}$ l'avvento del Cristianesimo porta alcuni cambiamenti nell'atteggiamento verso $i$ sogni erotici, in precedenza senza particolari connotati morali negativi, ${ }^{13}$ ma ora interpretati come possibili fautori di peccato; l'apparizione in sogno di donne seducenti fa parte delle prove a cui vengono sottoposti molti asceti e mistici cristiani. La preoccupazione del peccato carnale commesso in sogno trova espressione sia in forme popolari come l'inno Te lucis ante terminum:

\author{
Procul recedant somnia \\ et noctium phantasmata \\ hostemque nostrum comprime \\ ne polluantur corpora $[. .$.
}

sia nelle opere dei grandi pensatori cristiani come S. Agostino:

Iubes certe, ut contineam a concupiscentia carnis et concupiscentia oculorum et ambitione saeculi. [...] Sed adhuc vivunt in memoria mea, de qua multa locutus sum, talium rerum imagines, quas ibi consuetudo mea fixit, et occursantur mihi vigilanti quidem carentes viribus, in somnis autem non solum usque ad delectationem sed etiam usque ad consensionem factumque simillimum. Et tantum valet imaginis inlusio in anima mea in carne mea, ut dormienti falsa visa persuadeant quod vigilanti vera non possunt. ${ }^{14}$

Nonostante le riserve espresse nella letteratura cristiana, il sogno erotico appare occasionalmente nella lirica provenzale, servendo sia a delineare la condizione infelice dei protagonisti - questa volta maschili - e l'impossibilità di dare sfogo concreto ai loro desideri, sia ad accorciare la distanza tra poeta ed amata tipica dell'amor de lonh concedendo un piacere forte quanto fugace. ${ }^{\text {Is }}$ Il sogno erotico viene visto inoltre come un modo per originare e rafforzare il legame amoroso, come nel trattato sull'amor cortese di Andrea Cappellano: "Praeterea, si coamantem somnium repraesentat amanti, oritur inde amor et ortus sumit angmenta). ${ }^{.6}$ 


\section{Il sogno in Petrarca}

Il tema del sogno è ben presente nelle opere volgari del Petrarca, dove assume valenze diverse. Le parole 'sogno' e 'sonno' vengono utilizzati prevalentemente in senso metaforico per indicare la futilità e la fugacità della vita terrena o dell'amore sensuale. In tal senso vanno interpretati anche la maggior parte dei riferimenti a sogni 'reali' nelle rime in vita. Il sogno viene descritto come un nemico che "'l cor sottragge / a quel dolce penser che 'n vita il tene» ( $\operatorname{Rvf} 226,10-I I)$, in opposizione all'insonnia amorosa e in linea con quanto avviene nell'elegia romana. Nonostante - o in virtù di - la sua qualità illusoria, il sogno si configura anche come l'unico stato in grado di conferire al poeta una qualche felicità; particolarmente importante in questo contesto è l'apertura di Rvf 212 "Beato in sogno et di languir contento / d'abbracciar l'ombre et seguir l'aura estivas. ${ }^{17}$ Sebbene il sonetto si riferisca in primo luogo agli inganni dell'amore e alla sua natura paradossale, sviluppata poi in una serie di impossibilia, vi si intravede un chiaro cenno sia alla possibilità di un appagamento onirico non concesso nello stato di veglia sia alla conclusione 'canonica' di molti incontri in sogni o visioni con la persona amata: il vano tentativo di abbracciare un fantasma. ${ }^{8}$ Tra le rime in cui si menziona il sogno o il sonno dobbiamo annoverare anche un'allusione al mito di Endimione, l'unico riferimento esplicitamente 'erotico' collegato al tema (ma si tratta solo di un auspicio) nel Canzoniere:

Deh or foss'io col vago de la luna adormentato in qua' che verdi boschi, et questa ch'anzi vespro a me fa sera, con essa et con Amor in quella piaggia sola venisse a starsi ivi una notte; e 'l dí si stesse e 'l sol sempre ne l'onde.

$$
(R v f 237,31-36)
$$

I connotati del sogno nel Canzoniere cambiano a partire dalle rime limitrofe alla sezione 'in morte': da questo punto i sogni descritti come 'realmente' accaduti sono in genere veritieri e diventano anche, specie dopo la morte di Laura, un canale privilegiato di comunicazione con il mondo ultraterreno. La funzione consolatoria delle visioni oniriche, sviluppata in modo esteso nelle rime in morte, viene esplicitata nell'aper- 
tura di $\operatorname{Rvf} 250$, «Solea lontana in sogno consolarme / con quella dolce angelica sua vista / madonna», che però si muta repentinamente in un presagio di morte. Nelle 'rime in morte' visioni e sogni si susseguono (Rvf 282-86; 302; 340-43; 356; 359; identici concetti in TM II), ${ }^{\mathrm{I}}$ con una duplice funzione consolante e salvifica. In questa seconda parte, il tema del sogno si gioca principalmente sul contrasto tra il dolore incessante per la morte di Laura e il momentaneo sollievo offerto dal suo fantasma; manca pertanto ogni dubbio circa la veridicità del sogno e di conseguenza il tema, costante nella lirica cinquentesca, dei suoi inganni. In prospettiva petrarchista va comunque notato che solo nelle descrizioni di sogni post mortem si allude ad un contatto fisico tra il poeta e l'amata, altrimenti non previsto dal codice petrarchesco: Laura si siede sul letto del Petrarca, gli tiene la mano, gli ascinga le lagrime dal viso e gli parla apertamente. La possibilità di una rilettura in chiave sensuale di tali visioni è evidente, ad esempio, nella poesia latina del Pontano, che rivive in sogno gli amplessi con la defunta moglie Arianna:
Umbra sis felix mihi; suntne veri, uxor, amplexus? Vigilantis anne cura te in somnis agit, atque vana ludis imago? Umbra sed quamvis mihi cara, salve, et mihi felix ades; osculantem osculans tete accipioque, amansque am- plector amantem. ${ }^{20}$

Una versione più morigerata si legge in un sonetto di Berardino Rota dove lo sfondo erotico viene indicato soprattutto dalla citazione properziana in apertura: ${ }^{2 \mathrm{I}}$

Candida notte e più che 'l dì serena, che 'l ben mi dai che già morte mi tolse; ahi, perché l'alma anchor teco non volse girsen col sonno e con sua dolce pena?

Ritorna, prego, e quel piacer rimena che dolcemente i miei spirti raccolse dispersi e vaghi, e nel partir poi sciolse in caldo vento, in lagrimosa vena.

$[\ldots]$ 
Che potea più la notte e 'l sonno darme?

O caro inganno! Il meglio i' taccio e celo:

resti pur la memoria a consolarme. ${ }^{22}$

\section{Il sogno erotico nel Cinquecento ${ }^{23}$}

Sulla scorta dei poeti provenzali, e della lirica latina e neolatina, il tema del sogno erotico si afferma nella poesia volgare a partire del Quattrocento. ${ }^{24} \mathrm{Nel}$ Cinquecento assistiamo ad una serie di significative novità rispetto alla declinazione del tema del sogno nel Canzoniere del Petrarca. La più importante è che si tratta quasi sempre di sogni 'in vita' dove l'elemento erotico diventa prioritario rispetto alla funzione salvifica affidata alle apparizioni di Laura. Come altri temi analoghi - il bacio, la notte d'amore - il sogno erotico introduce nella lirica petrarchista una sensualità pressoché assente nei Rvf, piegando a questa funzione concetti come la consolazione e il sollievo dalla sofferenza. Il sogno erotico rimane però - forse per la mancanza di un modello forte petrarchesco - un topos sostanzialmente 'parassitario' che si esprime attraverso elementi propri di altri temi più saldamente ancorati nella tradizione lirica: il ritratto della donna, l'alternanza tra dolore e felicità illusoria, le antitesi giorno/notte, luce/buio, vita/ morte. La mancanza di un modello unico di riferimento spiega anche la notevole variabilità metrica, stilistica, retorica e contenutistica di queste rime, che attingono ad un gran numero di fonti volgari, latine, greche e provenzali, operando uno scambio continuo di motivi ed immagini con la poesia umanistica. ${ }^{25}$ Alcune rime sul sogno erotico si inseriscono all'interno di canzonieri o raccolte strutturate (gli esempi più significativi si leggono nelle rime del Cariteo, ${ }^{26}$ del Sannazaro ${ }^{27}$ e del Bembo ${ }^{28}$ con vere e proprie microsequenze sul tema); più spesso circolano in forma spicciola, veicolate dalle antologie liriche. Nelle pagine seguenti dettaglieremo alcuni dei motivi ricorrenti nelle rime cinquecentesche sul sogno erotico, evidenziando punti di contatto e differenze con la poesia umanistica, che rappresenta il principale interlocutore per questo tema.

\section{La piacevolezza del sogno}

Piuttosto che a descrizioni realistiche e dettagliate, il contenuto sensuale dei sogni viene affidato spesso a generiche allusioni alla sua piacevol- 
ezza, al punto che diventa difficile distinguere nettamente tra liriche erotiche, rime in cui si gioca sull' ambiguità di termini come 'dolcezza' $e$ 'piacere, ${ }^{29}$ e quelle in cui appare l'amata - o l'amato - con funzione esclusivamente consolatoria. Tipico in questo senso è un madrigale di Gaspara Stampa, in cui solo vaghe suggestioni concorrono a suggerire che si tratti di un sogno erotico:

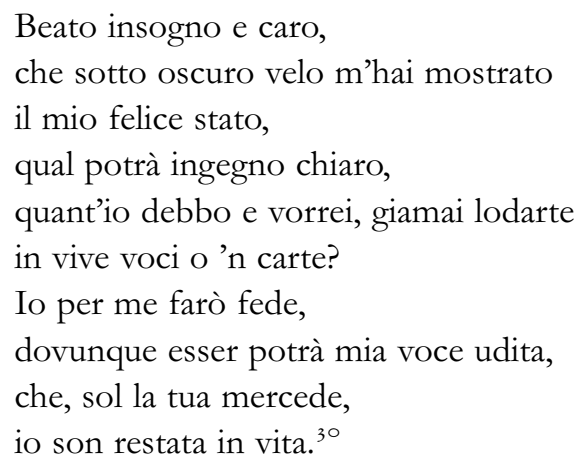

Per la sua dolcezza il vissuto onirico si pone in diretto contrasto con l'esperienza diurna:

Spesso a guisa d'un caro e fido amico da un dolce sogno visitato i' sono, e di notte colei m'apporta in duono per cui di pianto il giorno i' mi nudrisco. ${ }^{31}$

La piacevolezza del sogno viene comunicata anche attraverso una nutrita serie di espedienti: il lamento per la sua brevità:

c'al mondo or non sarebbe uom sì contento, se non fosse il mio ben stato sì breve. ${ }^{32}$

l'invettiva contro l'aurora che lo interrompe, riprendendo un motivo elegiaco: ${ }^{33}$

Ai Febo, se già in questi ameni campi, in questi verdi colli e fresche rive, pastor amasti pur terrene dive, perché tronchi il mio ben coi fieri lampi?34

il desiderio che il sogno si prolunghi: 
Intanto il sonno si partia pian piano; ond'io, per ingannarme, lungo spazio non volsi gli occhi aprire; ma da la bianca mano, che sì stretta tenea, senti' lasciarme. ${ }^{35}$

o che ritorni presto:

Beato se', ch'altrui beato fai: se non ch'usi troppo ale al dipartire, e 'n poca ora mi tôi quel che mi dai. Almen ritorna, e già che 'l camin sai, fammi talor di quel piacer sentire, che senza te non spero sentir mai. ${ }^{36}$

\section{L'antitesi giorno/notte}

Sfruttando una delle antitesi più trite della tradizione petrarchesca e al contempo il topos della notte luminosa mutuato da Properzio, ${ }^{37}$ molti componimenti associano la notte, grazie alla visione della donna concessa dal sogno, con la luce e il giorno; per converso il giorno, dovuto all'assenza della donna, diventa tenebroso. Questa declinazione del tema è particolarmente presente nella raccolta del Cariteo, in cui-come si desume dal titolo Endimione - il poeta si identifica con il mitico pastore e la donna con la luna:

Ma poi che ricomincia uscire il sole, et col novo splendor n'adduce il giorno, sì presto fugge il lume de la Luna, ch'io resto oscuro, quando gli altri han luce; tanto riposo, quanto dura il sonno, et per me il dì sereno è negra notte. ${ }^{38}$

Lo stesso concetto si legge in Annibale Nozzolino, per il quale è l'aurora che, risvegliando il poeta, porta l'oscurità:

Quelle tenebre a me fur luce e quella luce oscura mi fu, che 'l mio ben seco tolse, e me desto fe' contr'a mia voglia. ${ }^{39}$ 
Che si tratta di un motivo molto diffuso viene confermato dalla sua presenza sia nella poesia umanistica precedente:

Nocte quidem, coniunx, tecum vagor et tua mecum umbra venit; sic nox luxque diesque mihi est.

Luce autem sine te tenebris obversor et ipse me sine sum; sic lux nox tenebraeque mihi est. O valeant luces, lateat sol; sic mihi, coniunx, vives, sic moriar vivus et ipse tibi..$^{\circ}$

sia in contesti più prosaici:

[...] la notturna quiete, che libera da corporali impedimenti il pensiero, e spregiona i sogni, ch'el dì teneva chiusi e stretti, con una dolce et amorosa visione mi reca innanzi il dolce lume che la mente mia rasserena, e di notte oscura mi fa chiaro giorno, poi quando l'alba desta i miseri mortali, et alle diurne fatiche li chiama, insieme con la disiata visione si diparte, et io lasso senza luce mi rimango. Così di questi duo contrarii tempi l'uno è il mio conforto, e la mia vita, l'altro è la doglia e la morte mia. E parmi amore per far più lungo stratio del tormentoso mio cuore, mi presti haver sì liete, e tranquille, e serene notti, perché se quel vagho e dolce errore con l'imaginata luce del sole non rischiarasse le tenebre della mente, ne ristorasse l'afflitta e più tosto perduta vita; io havrei chiusi già gli occhi e spento il cuore in sempiterna notte. ${ }^{41}$

\section{Il paradosso insito nel concetto viene illustrato efficacemente dal Tansillo:}

E se dal lungo pianto ora m'avanza, il sonno in braccio per pietà mi renda la bella, cara, angelica sembianza. ${ }^{42}$

Ma questo, ohimè, tem'io ch'invan s'attenda:

come il sonno, amator de le fredd'ombre, portar può cosa che tant'arda e splenda? ${ }^{43}$

\section{Il sogno come 'premio', il premio sognato}

Numerosi componimenti presentano l'amore ricevuto in sogno come un premio per le sofferenze del poeta, spesso seguendo un copione pressoché identico: la donna appare in sogno al poeta mostrandosi meno crudele del solito; il poeta prende coraggio e le confida il suo amore e le sue sofferenze; la donna si impietosisce e gli si offre spontaneamente come 
'guiderdone' per la sua fedeltà; l'incontro viene interrotto da un brusco risveglio:

Ahi, chi mi rompe il sonno? Ahi, chi mi priva, misero, di quel ben ch'ogn'altro avanza?

Chi mi leva di man quella speranza

ch'era già, lasso, pur condotta a riva?

Era meco Madonna hor ch'io dormiva

e sì dolce m'apparve a la sembianza

che di seco parlar preso baldanza

i miei chiusi pensier tutti le apriva.

Di ch'ella mossa: «in guiderdon di questa

tua fede, in premio di cotanto amore, eccomi», disse, «a le tue voglie presta».

Ahi, che mentre l'abbraccio e pien d'ardore

la stringo, invido il sol ratto mi desta, che, ferendomi gli occhi, uccise il core. ${ }^{44}$

In altri casi è il sogno stesso ad essere il premio, dando al poeta ciò che non viene concesso nella realtà:

Quel che haver non potei, lasso, per via de' preghi o per virtù di tragger guai, né per forza di lagrime giamai, da la fiera d'Amor nemica e mia, un dolce sogno, e sì soave, pria che di Titon la sposa aprisse i rai, mi strinse al seno, ed ella non fu mai sì cruda, com'a l'hor benigna e pia. ${ }^{45}$

Più insolito è il sonetto $\mathrm{O}$ notte, o vision dolce e gioiosa di Angelo di Costanzo che - caso raro - sfiora l'oscenità, in cui la comparsa della donna in sogno segue una richiesta alla dea Venere:

Ier sera dissi: «O santa e gloriosa

dea, che dal vasto mar origin hai

e regni al terzo ciel, che fin darai

a questa nova mia fiamma amorosa?»

Poi giunse 'l sonno; et ecco in mezo un nembo

di vaghi fior la bella dea m'apparve,

e collocommi il mio tesoro in grembo; ${ }^{46}$ 
lo qual, più al vero ch'a fallaci larve simile, entrar lasciò nel porto il lembo, e con sommo piacer poscia disparve. ${ }^{47}$

Una variante sul motivo del premio è quella, appena abbozzata da Ovidio, della violenza onirica: il sogno erotico concede al poeta di godere della donna contro la sua volontà, vendicandosi della sua crudeltà:

tal che, pensando e desiando, io manco, qual vidi e strinsi quella man gentile, e qual vendetta fei del velo bianco. ${ }^{48}$

Il motivo compare anche nel Navagero, ancora a conclusione di un componimento sul sogno erotico:

At tu, proterva, quolibet fuge, eripe complexibus te te meis:

si somnus iste me frequens reviserit, tenebo te, invitam licet.

Quin dura sis, sis quamlibet ferox: eris et mitis, et facilis tamen. ${ }^{49}$

\section{Il catalogo di bellezze}

Il tema del sogno erotico funge spesso da pretesto per fornire un ritratto della donna che si spinge oltre il canonico elenco di bellezze di stampo petrarchesco (capelli, occhi, bocca, seno coperto) verso una maggiore sensualità. ${ }^{\text {so }}$ Se il Sannazaro ci offre un catalogo rigorosamente petrarchesco (configurandosi dunque come sogno amoroso più che erotico) $e$ presentato con formula dubitativa in omaggio alla canzone onirica del Petrarca nel sonetto Son questi i bei crin d'oro, onde m'avinse $(6 I),{ }^{5 \mathrm{I}}$ più frequentemente $i$ poeti si focalizzano su parti specifiche del corpo della donna come il seno nudo o il piede:

Dormiami, e si dormiva

agli occhi miei non pur la mia guerriera,

ma né punto anco fera:

ond'io pur l'assaliva:

era Amor meco, e 'l piede hor le scopriva, or quel candido seno; oh latte, oh neve; ma troppo oimè fu 'l sonno a sparir lieve. ${ }^{52}$ 
e talvolta velate allusioni alle parti intime della donna:

O quai labri baciai, quai cose vidi non mi lice parlar, che 'l dolce ancora (così vergogna vuol) si tien celato. ${ }^{53}$

In alternativa, l'erotismo può scaturire dal fatto che al poeta viene concesso di contemplare ogni parte del corpo della donna, e dalle sue reazioni, come in un sonetto di Girolamo Aquaviva in cui l'anima del poeta dormiente si stacca dal suo corpo per visitare l'amata, anch'essa addormentata:

Quivi la sua nemica in tempo assale che 'l dolce sonno le sue membra solve, et mentre attende et mira a parte a parte da l'aurea testa infino a i piedi eburni, trema di meraviglia e di dolcezza. ${ }^{54}$

A contraddistinguere gli elenchi sensuali non sono soltanto le parti corporee nominate o indicate attraverso perifrasi, ma la precisazione che il poeta li tocca o bacia, rompendo il tabù petrarchesco e neoplatonico del contatto fisico: 55

e fai ch'entro al bel seno

questa man lieta si rivolge e spiega, e perch'io goda a pieno

lasci ch'a' bianchi, alabastrini poggi

l'avida bocca ancor e i labbri appoggi. ${ }^{56}$

Occasionalmente viene precisato che la visione onirica rievoca un'esperienza reale:

Quest'è pur il bel piè, cui le sals'onde vezzosamente fiedono, che pria con più tenere labbia humil baciai.

Qual cruda, ahi, man dal sonno hor mi disvia, qual luce più che nube atra m'asconde di così grata vista i dolci rai? ${ }^{57}$

Più che alla tradizione volgare tali ritratti sensuali attingono alle descrizioni elegiache, come quella di Ovidio: 
Quos umeros, quales vidi tetigique lacertos!

Forma papillarum quam fuit apta premi!

Quam castigato planus sub pectore venter!

Quantum et quale latus! Quam iuvenale femur! ${ }^{8}$

già portati in ambito onirico dalla poesia umanistica:

O quales, quales mammas, o qualia crura, quale femur tetigi! Nil ego voce loquor.

O pudet, et quae sunt dulcissima iure silentur. ${ }^{59}$

Alcuni ritratti sono strutturati come una specie di trompe l'oeil letterario, in cui soltanto alla fine si rivela al poeta e al lettore che si tratta di un sogno, in coincidenza con il culmine del piacere. Questa formula gode di particolare fortuna tra i rimatori partenopei, fra cui il Sannazaro con il sonetto 6I e il Cariteo:

«Quest'è pur quella fronte alta et gioconda che turba et rasserena la mia mente; quest'è la bocca, che soavemente d'amorosa dolcezza hor mi circonda.

Questi son gli occhi che 'n la più profonda parte del cor m'han posto fiamma ardente; et questo è 'l petto che profusamente d'almo candore et pudicitia abonda.

Hor ne le braccia io tengo il corpo adorno d' ogni valore, hor son con la mia dea, hor mi concede Amor lieta vittoria...».

Così parlar dormendo mi parea; ma poi che gli occhi apersi et vidi il giorno, in ombra si converse ogni mia gloria. ${ }^{60}$

Lo stesso espediente viene adottato dal Tansillo nella sua canzone onirica, a conclusione di una lunga descriptio mulieris:

Sostien, vita, ch'io scopra

il nascoso tesoro

che mi fe' ricco andar sol del desire:

e de la nobil opra

non pur le gemme e l'oro

ma il terso e molle avorio lieto ammire. 
Cor mio, perché t'adire?

Perché turbi il bel viso, ch'or ora era sì chiaro?

Già il rasserena, o caro

mio bene: u' sono, in terra o in paradiso?

Oimé, ch'io moro! Or quanto

fia dolce il mezo, s'il principio è tanto?

Ah, gallo iniquo e fiero,

che col funebre strido

il sonno insieme e le mie gioie hai rotte! ${ }^{6 \mathrm{I}}$

\section{Il bacio sognato ${ }^{62}$}

Sulla scorta della poesia umanistica alcune rime sul sogno erotico si focalizzano su un aspetto particolare: il bacio. In linea con altre declinazioni del tema, mancano sia l'interpretazione neoplatonizzante del bacio come momento di comunione tra due anime, ${ }^{63}$ sia le descrizioni dettagliate e realistiche di alcuni componimenti latini (nonché delle successive rivisitazioni barocche); il contrasto risulta evidente ponendo a confronto il Pontano:

In somnis tenerum mihi labellum

offers dum male, suaviorque utrumque,

decursim lacrimae tibi exciderunt

et largo faciem madore tinguis;

atque has dum lacrimas madenti ab ore

detergo simul et simul relingo,

surreptim mihi mordicusque linguam

exceptam rapis obterisque dente $[\ldots]^{64}$

con la prova di Giulio Camillo sullo stesso tema:

Rugiadose dolcezze in matutini celesti humor che i boschi inargentate; dolci canne da noi tanto pregiate;

et voi doni de l'api alti et divini;

$[\ldots]$

in due labra dolcissime rosate

gustato ho i vostri alberghi pellegrini. ${ }^{65}$ 
Il sonetto si concentra su un singolo aspetto del bacio, la dolcezza, resa con una serie di metafore tradizionali, con solo un cenno finale ad un altro aspetto gradevole, il colore rosato delle labbra dell'amata. Il senso del gusto che nelle liriche sul bacio si contrappone alla vista e al tatto, predominanti nei ritratti, viene affiancato occasionalmente anche dall'olfatto, come in questo raro esempio di lirica del sogno erotico al femminile:

\author{
E appressandomi il viso \\ mi porse infra rubin due fresche rose \\ non mai ne l'odorifero oriente \\ viste più belle, o là su in paradiso, \\ in cui soavità tanta il ciel pose, \\ che altra maggior fra noi qui non si sente, \\ la cui sì bella vista \\ e 'l disusato odore \\ tornò subito al core \\ la smarrit'alma sconsolata e trista: \\ cose ch'a pena in ciel veder si ponno. \\ Deh, perché non fu eterno un sì bel sonno? ${ }^{66}$
}

Gli inganni del sogno e il dubbio se il sogno sia vero o falso

Nonostante la tradizionale insignificanza dei sogni erotici, molti poeti si interrogano sulla veridicità delle loro esperienze oniriche, auspicandosi che il sogno si riveli profetico ${ }^{67}$ o lamentandosi dei suoi inganni. Spesso questo desiderio si esprime in modo indiretto, con la precisazione che il sogno ha avuto luogo all' alba, il momento piu propizio per $i$ sogni veri. Cosi Giulio Camillo puntualizza che ha sognato di baciare la sua donna «tra gli oscuri e $i$ lucidi confini / de la notte et del dì mentre Camillo Pellegrino situa il suo sogno erotico appena prima dell'alba:
Era già per lasciar freddo nel letto
il suo vecchio Titon la bella Aurora
quando 'l volto gentil, che m'innamora,
m'apparse in sogno con ridente aspetto. ${ }^{68}$

Talvolta sussiste il dubbio se il sognante sia sveglio o addormentato, come in questo sonetto pastorale di Lodovico Dolce: 
Ei, che tanta beltà veder non suole, in dubbio s'egli dorme o s'egli è desto, prestando a' suoi piacer felice via: «Fa'», dice, «O bella Dea, se sonno è questo, ch'io sempre chiuda gli occhi e 'l chiaro sole mai non giunga a turbar la notte mia». ${ }^{69}$

traduzione abbastanza fedele di parte di un componimento latino di Johannes Secundus:

Iulia te teneo: superi, teneatis Olympum.

Quid loquor? An vere, Iulia, te teneo?

Dormione? An vigilo? Vera haec? An somnia sunt haec?

Somnia seu sunt, seu vera, fruamur, age.

Somnia si sunt haec, durent haec somnia longum, nec vigilem faciat me, precor, ulla dies. ${ }^{70}$

Se la falsità dei sogni erotici èpacifica, però, la reazione del poeta davanti a questa constatazione varia notevolmente. In alcuni casi la piacevolezza del sogno falso, una volta svanita, porta ulteriori sofferenze:

Appena il sonno ai membri miei s'avventa

ch'al cor doglioso e sol d'amore armato

un falsissimo error mi rappresenta, e mi dimostra il bel sembiante amato perché privo di quello io veggia e senta tanto più il danno e 'l mio doglioso stato. ${ }^{71}$

Per altri rimatori, invece, gli inganni del sogno sono graditi, purché ritornino:

Ah sonno disleal, pieno d'inganno, quando il bel viso mi recasti in dono, a che finger amor, se sdegno v'era?

Tornami ad ingannar, ch'io te 'l perdono, sonno, poi che bisogna per men danno cercar l'ombra e fuggir l'effige vera. ${ }^{72}$

Infine, in casi eccezionali gli auspici del poeta si avverano, come nel dittico di sonetti di Petronio Barbati: nel primo il poeta si domanda se la visione dell'amata sia vera o falsa, per scoprire al risveglio che è falsa; 
nel secondo, dopo aver espresso una serie di dubbi sul sogno, il poeta si sveglia e si rende conto che ciò di cui ha sognato è reale:

$\mathrm{O}$ ciel benigno, o me più che beato!

Ecco, ch'io son pur desto, egli è pur desso,

e pure il vedo, il bascio, il tocco e stringo! ${ }^{73}$

\section{Il mito di Endimione e il desiderio di morte}

Il tema del sogno erotico è spesso associato alla figura mitologica di Endimione, il bellissimo pastore addormentato in eterno dalla Luna e da lei amato in sogno. Nonostante le numerose interpretazioni neoplatoniche, mistiche ed ermetiche del mito, che veniva anche utilizzato come metafora per la creazione artistica e letteraria, ${ }^{74}$ la tradizione lirica volgare si concentra quasi esclusivamente su due aspetti: il contrasto tra la felice sorte del giovane, a cui viene concesso di godere in eterno della sua amata, e le pene senza fine del poeta innamorato; il desiderio, pur di condividere questa sorte, di dormire in eterno e quindi di morire.

Nella letteratura classica l'associazione di Endimione con la morte e la parentela tra sonno/sogno e morte 75 viene interpretata prevalentemente in chiave negativa, come antitesi di amore e vita, come si evince chiaramente da Ovidio:

Infelix, tota quicumque quiescere nocte sustinet, et somnos praemia magna vocat!

Stulte, quid est somnus gelidae nisi mortis imago?

Longa quiescendi tempora fata dabunt. ${ }^{76}$

Tale prospettiva cambia nella poesia umanistica, dove la sorte di Endimione - e di conseguenza la morte - viene vista spesso come auspicabile, in quanto capace di alleviare $i$ tormenti e le insonnie dell'innamorato. Il tema viene declinato con modalità diverse: al Landino è preclusa perfino la possibilità di sognare la sua amata, mentre ad Endimione, placidamente addormentato, viene concesso di godere in eterno della sua dea:

Felix Endymion, viridi quem mollis in herba

pressit et evinxit lumina fessa sopor.

Ah quotiens illi gelida sub rupe iacenti

incubuit tepido Cynthia pulchra sinu, 
et modo formosis puerum complexa lacertis carpebat niveis oscula grata genis, et modo dissimili pingebat tempora flore puniceis nectens lilia cana rosis.

His me, vel falsis, potuisses, perfide somne, nam quoque falsa iuvant - ludere imaginibus. ${ }^{77}$

Per il Poliziano, invece, è proprio un sogno erotico a svelare al poeta la fortunata sorte di Endimione, rendendo auspicabile pure la morte e ribaltando la prospettiva ovidiana:

O mihi quanta datis fallaces gaudia Somni!

Invideo, Endymion, Latmia saxa tibi.

Iam si nil sopor est gelidae nisi mortis imago, omnia mors superat gaudia: vita vale. ${ }^{78}$

Gli stessi concetti appaiono con frequenza nella lirica volgare; cosi il Cariteo chiede di morire per continuare a sognare:

Morir vorrei dormendo eternamente;

ché, se 'l somno a la morte è somigliato, in tal morte io vivrei felicemente. ${ }^{79}$

Il Sannazaro riprende la struttura bipartita già adoperata dal Landino per sottolineare il contrasto tra le gioie eterne di Endimione e quelle troppo brevi del poeta:

Felice Endimion, che la sua diva, sognando, sì gran tempo in braccio tenne, e più, se al destar poi non gli fu schiva! $!^{80}$ Ché se d'un'ombra incerta e fuggitiva tal dolcezza in un punto al cor mi venne, qual sarebbe ora averla vera e viva? ${ }^{81}$

La parentela tra sonno e morte consente ai poeti di sviluppare una serie di paradossi che scaturiscono dall'associazione della donna con la vita: così è il sonno, parente della morte, a dare al poeta la vita, nella forma delle visioni oniriche dell'amata:

Giacea la mia virtù vinta e smarrita dal duolo in sua ragion sempre più forte, 
quando il sonno pietoso di mia sorte seco addusse madonna a darle aita; che sollevò gli spirti, e 'n me sopita la doglia, a nove speme aprìo le porte. Così allor ne l'imagine di morte trovò l'egro mio cor salute e vita. ${ }^{82}$

$N e l$ De rerum natura, trattando delle fantasie sessuali degli adolescenti, Lucrezio rovescia la prospettiva tradizionale secondo cui $i$ sogni erotici sono soltanto vani prodotti dell'immaginazione, accostando invece le illusioni del sogno a quelle del desiderio carnale in generale (IV I030 ss.). Questa lettura, secondo cui il sogno erotico diventa un microcosmo dell'amore, si presta perfettamente ad un'interpretazione del tema nella lirica petrarchista del Cinquecento. Nonostante il notevole apporto di novità a livello microtestuale, infatti, il sogno erotico rimane essenzialmente una variante sul tema della temporanea sospensione delle pene, senza offrire nuovi sbocchi narrativi o spingere verso una diversa concezione dell'amore. Lontani dalla sensualità gioiosa e spensierata di tanta lirica latina e neolatina, e al contempo privi dei significati platonizzanti o spirituali talvolta presenti nelle visioni oniriche del Quattrocento, $i$ sogni amorosi dei lirici volgari del Cinquecento rimangono confinati nell'universo chiuso del petrarchismo, offrendo al massimo una breve finestra su una felicità impossibile: un'ulteriore conferma «che quanto piace al mondo è breve sogno».

Erika Milburn 
Ringrazio Franco Tomasi per i suoi preziosi consigli e per l'invito a contribuire a questo numero della rivista.

i. Omero, Od. xix 560-67; Virgilio, Aen. vi 893-96.

2. Il luogo canonico è ovviamente Sofocle, Edipo re 98 I-82 ma cfr. ad esempio il sogno di Cesare in Svetonio, Vita divi Iuli, 7 che prefigura le conquiste militari; il motivo é già in Erodoto, Storie VI 107.

\section{Macrobio, Commentariorum in Somnium Scipionis I 3.}

4. Artemidoro, Il libro dei sogni, trad. Di D. Del Corno, Milano, Adelphi, I975: IV, praef. Sul libro di Artemidoro e in genere sul sogno nel pensiero tardo-antico cfr. P. Cox-Miller, Il sogno nella tarda antichità. Studi sullimmaginazione di una cultura, Roma, Jouvence, 2004 (ed. orig. Dreams in Late Antiquity. Studies in the Imagination of a Culture, Princeton University Press, 1994), in part. pp. 90-103.

5. Sul sogno erotico nella Grecia antica cfr. J. Pigeaud, Il sogno erotico nell'antichità greco-romana: l'oneirogmos in Il sogno in Grecia, a cura di G. Guidorizzi, Bari, Laterza, I 988 , pp. I $37-46$.

6. Cfr. J. Bouquet, La nuit, le sommeil et le songe chę les élégiaques latins, in «Revue des études latines», 74 (1996), pp. I 82-2 I I.

7. Cfr. Ovidio, Am. i i 3; Tibullo, Eleg. i 2, 73-76.

8. Per il sonno del marito che favorisce l'amante cfr. Ovidio, $A m$. I 4, 53-54; I 9, 25-26; Tibullo, Eleg. I 6, 27-28.

9. Tra le poche eccezioni i cenni ad un sognante mashile in Orazio, Carm. IV I, 37-40; Virgilio, Buc. vili io8. Cfr. anche Eschilo, Agamennone 420-26.

Io. Su questo aspetto cfr. C. Garriga, Somnis eròtics: entre el mite i la fisiologia, in Estudis de llengua i literatura catalanes /XXI Miscel-lània Joan Bastardas IV, Barcelona, Publicacions de l'Abadia de Montserrat I990, pp. 5-I4.

i lumina cum placido victa sopore iacent».

I 2. Sul sogno nella tarda antichità e nel medioevo cfr. S. F. KRUGER, Il sogno nel medioevo, Milano, Vita e pensiero, 1996 (ed. orig. Dreaming in the Middle Ages, Cambridge, CUP, 1993); J. Амат, Songes et visions. L'au delà dans la littérature latine tardive, Parigi, Études Augustiniennes, I 985 ; I sogni nel medioevo, a cura di T. Gregory, Roma, Edizioni dell'Ateneo, i 98 ; P. C. Miller, cit.

I 3. Un'eccezione è il Platone della Repubblica, per cui i sogni erotici sono sintomi di un disturbo psichico, dell'incapacità di controllare i propri appetiti (IX $57 \mathrm{IC}$ ).

I4. In Confessions a cura di J. J. O’Donnell, Oxford, Clarendon Press, i992: x 30, 4I. Sul sogno in S. Agostino cfr. M. Dulaey, Le rêve dans la vie et la pensée de Saint Augustin, Paris, Études Augustiniennes, 1973. 
I 5 . Sul sogno erotico nella lirica provenzale cfr. H. BRAET, Visio Amoris. Génèse et signification d'un thème de la poésie provençale, in Mélanges d'histoire littéraire, de linguistique et de philologie romanes offerts à Charles Rostaing, Liegi, Université de Liège, I974, t. I, pp. 89-99.

I6. Andrea Cappellano, Trattato d'Amore, a cura di S. Battaglia, Roma, Perrella, I 947 , II 2.

17. Agisce anche il ricordo di Sir 34, I-3: «Vana spes et mendacium insensato viro et somnia extollunt inprudentes quasi qui adprehendit umbram et persequitur ventum sic et qui adtendit ad visa mendacia hoc secundum hoc visio somniorum ante faciem hominis similitudo hominis»; cfr. S. CHEssa, Il profumo del sacro nel Canzoniere di Petrarca, Firenze, Società Editrice Fiorentina, 2005, pp. 99-103.

I 8. Il motivo è diffusissimo; tra gli esempi più famosi: il tentativo di Achille di abbracciare Patroclo che conclude il loro incontro (Il. xxIII 99-207); quello di Odisseo di abbracciare la madre Anticlea (Od. xI 204-208); Creusa sfugge tre volte all'abbraccio di Enea (Aen. II 792-94); in ambito volgare cfr. Purg. II 79-8 I.

19. Molto diverso il racconto delle apparizioni di Laura in Epystole metrice I 6 (in F. Petrarca, Rime, Trionfi e poesie latine, a cura di F. Neri, G. Martellotti, E. Bianchi, N. Sapegno, Milano-Napoli, Ricciardi, I95 I), dove vengono descritte come veri e propri incubi.

20. Lyra Ix "Uxorem in somnis alloquitur", $\mathrm{I}-9$ in G. G. Pontano, Carmina, a cura di J. Oeschger, Bari, Laterza, I 948.

2 г. Properzio, Eleg. II I 5, I: «O me felicem! O nox mihi candida! [...]».

22. Bernardino Rota, Rime, a cura di L. Milite, Parma, Guanda, 200: son. I 89, I-8; I 2-I 4 .

23. Cfr. l'interessante studio di S. Carrai, Ad Somnum. L'invocazione al sonno nella lirica italiana, Padova, Antenore, I990 (in particolare sulla variante erotica pp. 94-I04); in ambito spagnolo C. MAurer, "Soñé que te... ¿dirélo?: el soneto del sueño erótico en los siglos xvi y xvii, in «Edad de Oro», 9 (I 990), pp. I 49-67 e A. Alatorre, El sueño erótico en la poesía española de los siglos de oro, Città del Messico, Fondo de Cultura Económica, 2003; meno utile invece G. P. Maragoni, "Sogni e copule io fingo". Avventure secentesche del Petrarca onirico, in Petrarca in Barocco. Cantieri petrarchistici, a c. di A. Quondam, Roma, Bulzoni, 2004, pp. 275-88.

24. Per un elenco di rime volgari sul tema cfr. F. Gandolfo, Il dolce tempo. Mistica, ermetismo e sogno nel Cinquecento, Roma, Bulzoni, 1978, pp. 49-53; si tratta generalmente di sogni amorosi e non erotici.

25. Per il tema del sogno nella poesia umanistica cfr. l'antologia di B. Windau, Somnus. Neulateinische Dichtung an und über den Schlaf. Studien zur Motivik. Texte, Übersetzung, Kommentar, Wissenschaftlicher Verlag Trier, 1998.

26. Benedetto Gareth detto il Chariteo, Le rime secondo le due stampe originali, con introduzione e note di E. Pèrcopo, Napoli, Tipografia dell'Accademia delle 
Scienze, i 892, sonn. i4-16, sestina i; sul Cariteo cfr. B. BArbiellini Amidei, Alla Luna. Saggio sulla poesia del Cariteo, Firenze, La Nuova Italia (sul sogno pp. 67-72; I 35-36).

27. Jacopo Sannazaro, Opere volgari, a cura di A. Mauro, Bari, Laterza, ig6r: Sonetti e canzoni 6o-69 (otto sonetti e due madrigali; la sequenza è prefigurata dal son. 58 , una preghiera ad Eolo affinché impedisca la partenza della donna, e dalla canz. 59 sulle sofferenze causate dalla sua assenza).

28. Pietro Bembo, Prose della Volgar Lingua. Gli Asolani. Rime, a cura di C. Dionisotti, Milano, Tea, I993: Rime 88-90. Nel caso del Bembo sembra che i sonetti furono ispirati da una vicenda reale, come si desume dal commento di Dionisotti, citando una lettera scritta dal poeta nel i 503 a Lucrezia Borgia: «[...] Dappoiché io a V.S. non scrissi, ho fatto sopra un cortesissimo e dolcissimo sogno d'una di queste passate notti tre Sonetti, i quali, perché sono ancora male rassettati, mi riservo a mandarvi un altro giorno insieme con qualche ritrovamento per le vostre scritture».

29. Per il significato erotico della 'dolcezza' cfr. J. Toscan, Le carneval du langage. Le lexique érotique des poètes de l'equivoque de Burchiello à Marino (XV-XVII siècles), Lille, Presses Universitaires de Lille, I 98 I, t. 2 pp. 805-806; per 'piacere' t. I, pp. 5 I 4- I6.

30. Gaspara Stampa, Rime, introduzione di M. Bellonci, note di R. Ceriello, Milano, Rizzoli, I994, madr. 233.

3 г. Girolamo Britonio, Gelosia del sole, Napoli, Mair, i 5 I 9, c. I 7 or.

32. Sannazaro, Sonetti e canzoni 63, 7-8. Identico concetto in Angelo Colocci, "Ad Somnum” I4-I 8: «[...] quasi si forem beatus, / si non somnia tam cito et fugaci / gressu evanida in antra convolassent / ubi et Cymmerii tui recessus / et solis radiis negata tellus» (in Poesie italiane e latine, Jesi, Bonelli, I 772).

33. Ad esempio Ovidio, $A m$. I I 3.

34. Matteo Montenero in Rime di diversi signori napolitani e d'altri. Libro settimo, p. I6.

35. Sannazaro, Sonetti e canzoni 64, I I-I 5.

36. Bembo, Rime 88, 9-I4.

37. Cfr. M. Malinverni, Per una notte luminosa: fortuna di un topos da Properzio ad Ariosto in Fra Satire e Rime ariostesche a cura di C. Berra, Milano, Cisalpino, 2000, pp. 499-5 I3.

38. Cariteo, Endimione, sest. I, 7-I 2.

39. Citato in Carrai, Ad Somnum cit., pp. I77-79.

40. Pontano, De tumulis II 60 "Pontanus uxorem Ariadnam in somnis alloquitur".

4I. Antonio Minturno, Lettere, Venezia, Scoto, I 549, lib. VII lettera 17 (cit. in GANDOLFO, Il dolce tempo, p. 50). 
42. Per l'immagine cfr. Pontano, Eridanus I 7, 37-39: «[...] sopor ultima praebet / gaudia, teque meo collocat ipse sinu, amplectorque, tuis innexus et ipse lacertis».

43. Luigi Tansillo, Rime, Introduzione e testo di T. R. Toscano, commento di E. Milburn e R. Pestarino, Roma, Bulzoni, 20 I I, p. 70.

44. Domenico Venier in Il sesto libro delle rime di diversi eccellenti autori, Venezia, al segno del Pozzo, I 553 , p. I 29v.

45. Tommaso Machiavelli in Libro quarto delle rime di diversi eccellentissimi autori, Bologna, Giaccarello, i 5 I, p. 5 I.

46. Venere che consegna l'amata al poeta in sogno è già in Johannes Secundus, Elegiae I Io, 5: «misit in amplexus illam Venus aurea nostros» (in P. Murgatroyd, The Amatory Elegies of Johannes Secundus, Leiden/Boston/Koln, Brill, 2000).

47. In S. Longhi, Una raccolta di rime di Angelo di Costanzo, in «Rinascimento», 2 a serie, I 5 (I975), pp. 23 I-90, son. I 8 . Il sonetto risulta inedito durante il Cinquecento.

48. Sannazaro, Sonetti e canzoni 62, I 2-I 4.

49. Andrea Navagero, Lusus, a cura di A. E. Wilson, Niuwkoop, De Graaf, I 973 , 29, $17-22$.

50. Sui ritratti femminili nella lirica cinquecentesca cfr. G. Pozzi, Il ritratto della donna nella poesia d'inizio Cinquecento e la pittura di Giorgione, in «Lettere italiane », 3 I ( 1979 ), pp. 3-30; E. RAImondi, Ritrattistica dell'eros, in I sentieri del lettore, a cura di A. Battistini, Bologna, il Mulino, I994, t. I, pp. I07-3 I; A. Quondam, Il naso di Laura. Lingua e poesia lirica nella tradizione del Classicismo, Modena, Panini, I991, pp. 291328; G. Forni, La "belle matineuse" e la ritrattistica dell'eros in La lirica del Cinquecento. Seminario di studi in memoria di Cesare Bozzetti, a cura di R. Cremante, Alessandria, Edizioni dell'Orso, 2004, pp. I07-22.

5 I. Rvf 359, 56-58: «Son questi i capei biondi, et l'aureo nodo / - dich'io ch'anchor mi stringe, et quei belli occhi / che fur mio sol?' [...]».

52. Giovan Battista Strozzi il giovane, Madrigali, a cura di L. Sorrento, Strasburgo, Heitz, I 909, 71, 9, 5-7.

53. Annibale Nozzolino, cit. in Carrai, Ad Somnum cit., pp. I77-79.

54. Giovanni Girolamo Acquaviva, son. Quando la notte spande le grandi ale in Rime di diversi [...] Libro settimo, p. [16].

55. Cfr. Bembo, Asolani in 6; Castiglione, Cortegiano iv 62.

56. Madrigale anonimo cit. in CARraI, Ad Somnum cit., p. 95.

57. Cesare Pavesi, son. Io pur riveggio amata ninfa e bella in De le rime di diversi nobili poeti toscani, raccolte da M. Dionigi Atanagi, libro secondo, Venezia, Avanzo, i 565 , c. I69r. 
58. Am. I 5, I9-22.

59. Girolamo Angeriano, Erotopaegnion. A Trifling Book of Love, a cura di A. M. Wilson, Nieuwkoop, De Graaf, I 995 , I 3 I “Ad somnum", 7-9.

6o. Cariteo, Endimione, son. I 5 .

6r. Tansillo, Rime 256, 66-8 I.

62. Sul tema del bacio ( $\mathrm{ma}$ in ambito barocco) cfr. F. Guardiani, Oscula mariniana, in «Quaderni d'italianistica», I6 (1995), pp. 197-243; M. NoseDA, Il bacio di carta. La parabola di un topos tra rinascimento e barocco, in Thematologie des Kleinen, a c. di E. Marsch e G. Pozzi, Friburgo, Éditions universitaires, I 986, pp. 94-1 30; V. GuERCIO, Ancora sui baci di carta: Marino, Guarini, Fenaruolo, in «Studi italiani», I 2 (2000), pp. $5-47$.

63. Sul motivo cfr. F. Valencia, "Acoged blandamente mi suspiro": el beso de almas en la poesia petrarquista española del siglo XVI in "DICENDA. Cuadernos de Filología Hispánica», 26 (2008), pp. 259-89, sulla lirica spagnola ma con ampia rassegna della precedente letteratura italiana ed umanistica.

64. Hendecasyllabi II i "Ad Focillam". Il tema del bacio sognato è frequente nella lirica umanistica; cfr. ad esempio il citato componimento dell'Angeriano I I-I 2: «Lassi ambo postquam requievimus, oscula labris / mille illis data sunt, reddita mille meis»; o quello del Colocci ı-- I : «dum confundimus hinc et hinc vicissim / verba et basia veriora veris».

65. Giulio Camillo detto il Delminio, in Rime diverse di molti eccellentissimi auttori nuovamente raccolte. Libro primo, Venezia, Giolito, i 545, p. 6r. Cfr. Anche la descrizione del Navagero: «[...] quin mihi / mille obtulit sponte oscula, / oscula, quae Hymetti dulciora sint favis, / quae suaviora nectare» (Lusus 29, 7-I 2).

66. Chiara Matraini, madr. Era il mio sol venuto al mio languire in Rime di diversi [...] Libro settimo, p. 9 I.

67. Il motivo si trova in apertura del citato componimento del Navagero: «Beate somne, nocte qui hesterna mihi / tot attulisti gaudia, / utinam deorum rector ille caelitum / te e coetu eorum miserit, / quae saepius mortalibus vera assolent / mitti futuri nuntia».

68. In Rime di diversi [...] Libro settimo, p. 274.

69. Son. Dal suo bel cerchio fra' 'l cornuto armento, in Rime diverse [...] Libro primo, p. 3 I 3.

70. Elegiae i Iо, 25-30.

71. Diomede Carafa, son. Non so che ritrovar mi possa il cielo, in Rime di diversi [...] Libro settimo p. 6r.

72. Tansillo, Rime I78, 9-I4. 
73. Son. Deh cosi fusse ver, sì come ingombra, in Rime di diversi nobili huomini et eccellenti poeti nella lingua thoscana. Libro secondo, Venezia, Giolito, I 547, c. I $58 \mathrm{v}$.

74. Sulla ricezione del mito tra Quattro e Cinquecento cfr. Gandolfo, Il dolce tempo cit., in part. pp. 43-75; M. RuvOLDT, The Italian Renaissance Imagery of Inspiration: Metaphors of Sex, Sleep and Dream, Cambridge, CUP, 2004; N. AgApiou, Endymion au carrefour: la fortune littéraire et artistique du mythe d'Endymion à l'aube de l'ère moderne, Berlin, Gebr. Mann Verlag, 2005.

75. Cfr. ad esempio VirgiLio, Aen. vi 278: «consanguineus Leti sopon».

76. $A m$. II 9b, I 5-I 8 ; il contrasto tra amore e sonno è anche in $A m$. II Io, i9-20.

77. Cristoforo Landino, Xandra, II I I, I7-26 in Carmina omnia a cura di A. Perosa, Firenze, Olschki, I939.

78. Angelo Poliziano, Epigrammata rog "in somnos" in Poesie volgari inedite e poesie latine e greche edite e inedite, a cura di I. Del Lungo, Firenze, Barbèra, i 867.

79. Cariteo, Endimione, son. i6, i 2-I4.

8o. Per l'invocazione, oltre al componimento di Landino cfr. Pontano, Hendecassyllabi I 30, I 5: «Felix Endymion suopte somno».

8I. Sannazaro, Sonetti e canzoni 63, 9-I 4.

82. Torquato Tasso, Rime 'eteree', a cura di L. Caretti, Parma, Zara, I990, 24, I-8. 